\title{
Original Investigation \\ Nicotine withdrawal symptoms following a quit attempt: An ecological momentary assessment study among adolescents
}

\author{
Rinka M. P. Van Zundert, Emiel A. Boogerd, Ad A. Vermulst, \& Rutger C. M. E. Engels
}

\section{Abstract}

Introduction: The present study describes growth curves of withdrawal symptoms among 138 daily smoking adolescents before, during, and after a quit attempt.

Methods: Participants reported their levels of withdrawal symptoms (craving, negative affect, and hunger) three times a day over a period of 28 days: 1 week prior to and 3 weeks following a quit attempt.

Results: All withdrawal symptoms were quite stable at a relatively low level during the 5 days prior to the quit day. At Day 8, withdrawal symptoms (especially craving) increased substantially. A significant decrease in symptoms was visible during the week following the quit day, and within 2 weeks postquit, both abstinent and relapsed adolescents had reverted to levels comparable to those during the prequit period. The course over time for craving and hunger were best described by a quadratic term, and a linear model best suited negative affect. Individual intercepts and slopes of the growth curves were used to predict abstinence during the last week of the study and at the 2-month follow-up. Analyses revealed that higher levels of craving at the beginning of the prequit week and on the target quit day (intercepts) decreased the odds of being abstinent during the last week of the study. In addition, the quadratic term for hunger predicted abstinence during the last week. Finally, among all three symptoms, none of the growth model characteristics predicted abstinence at follow-up.

Discussion: The findings generally suggest that smoking cessation among daily smoking adolescents does not largely depend on how their withdrawal symptoms evolve over time after achieving abstinence.

\section{Introduction}

Although research on smoking has focused mainly on initiation and escalation of use, more recent research has been devoted to the area of adolescent smoking cessation. Teenagers experience great difficulties when attempting to refrain from smoking, and approximately $90 \%-95 \%$ of adolescents who make an unaided attempt to quit smoking will relapse (Mermelstein, 2003; Sussman, 2002). Participation in adolescent cessation programs does not seem to improve success rates (Garrison, Christakis, Ebel, Wiehe, \& Rivara, 2003; Leatherdale, 2006). More insight into the natural history of adolescent quit attempts is needed to tailor psychosocial or pharmacological treatments to this group (Mermelstein, 2003).

Studies among adults have demonstrated that withdrawal symptoms can predict failure to quit smoking (McCarthy, Piasecki, Fiore, \& Baker, 2006; Piasecki, Fiore, \& Baker, 1998). Withdrawal symptoms refer to a set of physical and mental discomforts that emerge when individuals abstain from smoking, such as cigarette craving, irritability, restlessness, insomnia, anxiety, depression, increased appetite, and poor concentration (see Hughes, 2007, for a review). Withdrawal symptoms typically increase strongly during the first week of deprivation, after which they revert gradually to a level that is the same as or lower than baseline levels (Hughes, 1992; Jorenby et al., 1996; Piasecki et al., 1998; for an exception, see Shiffman et al., 1997), and individual characteristics of the course of withdrawal symptoms over time can predict cessation outcomes in adults (McCarthy et al., 2006). Although we know that adolescents also experience withdrawal symptoms during smoking deprivation and that they report similar symptoms (Prokhorov, Hudmon, Cinciripini, \& Marani, 2005; Smith, Cavallo, McFetridge, Liss, \& Krishnan-Sarin, 2008), few studies have modeled the natural history of withdrawal in adolescents following a quit attempt and related the course of withdrawal over time to relapse outcomes.
Rinka M. P. Van Zundert, M.Sc., Behavioural Science Institute, Radboud University Nijmegen, The Netherlands

Emiel A. Boogerd, M.Sc., Behavioural Science Institute, Radboud University Nijmegen, The Netherlands

Ad A. Vermulst, Ph.D., Behavioural Science Institute, Radboud University Nijmegen, The Netherlands

Rutger C. M. E. Engels, Ph.D., Behavioural Science Institute, Radboud University Nijmegen, The Netherlands
Corresponding Author:

Rinka M. P. Van Zundert, M.Sc., Behavioural Science Institute, Radboud University, PO Box 9104, 6500 HE Nijmegen, The Netherlands. Telephone: +31-0031-24-3612816; Fax: +3100-3124-3612776; E-mail: r.vanzundert@pwo.ru.nl 
For several reasons, we cannot assume that neither the course of withdrawal symptoms over time nor its association with cessation outcomes as observed among adults are by definition identical for adolescents. First, adolescence is a developmental phase in which impulsivity, novelty seeking, and suboptimal decision making are considered to be normative traits (Chambers, Taylor, \& Potenza, 2003), and in which individuals are still in the process of maturing and developing selfreflective and introspective skills (Beyth-Marom, Fischhoff, Jacobs-Quadrel, \& Furby, 1991; Steinberg \& Cauffman, 1996). In addition, both hormonal processes and social, cognitive, and environmental influences that are uniquely associated with adolescence contribute to adolescents' moods being different from those of young children and adults (Buchanan, Eccles, \& Becker, 1992). Adolescents may therefore experience more variable and more intense moods, more variable energy levels, more restlessness, and more anxiety than individuals at other stages of development.

Given these characteristics, it has been postulated that the similarities in withdrawal symptoms displayed by adolescents and adults might be attributed to the phase of adolescence itself rather than to cessation (Prokhorov et al., 2005). Although Prokhorov et al. (2005) suggest that this is true to some extent (since individual withdrawal symptoms did not differentiate effectively between never-smokers and former light smokers), symptoms for former smokers were reported retrospectively and duration of time since cessation was not taken into account. However, the psychophysiological characteristics and states that typify adolescence might affect withdrawal in other ways. Negative affect among adults, for example, seems to diminish over time to levels lower than those reported prior to quitting among successful quitters, and to persist at equal or even higher levels among those who fail. Moreover, a fairly robust within-subject association was found between negative affect and smoking lapses after a period of abstinence among adults (Kassel, Stroud, $\&$ Paronis, 2003). Since adolescence is characterized by increased and more variable levels of negative affect, it is possible that cessation does not instigate substantial increases in negative affect or that, if it does, it might not necessarily provoke relapse among adolescents as they may be more used to (and thus more tolerant of) mood changes. This hypothesis is supported in part by preliminary findings from Smith et al. (2008), who assessed withdrawal symptoms weekly for 4 weeks among adolescent smokers participating in a pilot cessation intervention. They did not find symptoms of depressed mood, irritability, difficulty concentrating, appetite, sleep problems, and anxiety on the quit day to differ significantly from the baseline assessments. Craving and restlessness, however, increased significantly after cessation. In addition, none of the symptoms predicted the likelihood of lapse during the treatment, although this finding may have been the result of the small sample size. Nonetheless, there seem to be clear indications why the course of tobacco withdrawal symptoms over time and its association with cessation outcomes might differ between adolescents and adults.

In the present study, data were collected using ecological momentary assessment (EMA), which uses intensive repeated sampling to gather data on momentary states of individuals in real-time and real-world contexts (Shiffman, Stone, \& Hufford, 2008). Participants reported on their withdrawal symptoms and smoking behavior three times a day during 4 weeks: 1 week prior to the quit attempt and 3 weeks after the quit attempt. The use of daily measures of withdrawal has two major advantages over retrospective ratings (especially those with large time intervals between the targeted experience and time of reporting). It reduces the susceptibility of reports to recall bias and it provides sufficient data to be able to assess within-person developmental processes (Shiffman et al., 2008).

The present study describes the elevation and shape of the growth curves of the withdrawal symptoms craving, negative affect, and hunger during the periods preceding and following a target quit date in a large sample of 138 adolescent daily smokers. We also tested whether individual growth curve estimates differed as a function of postquit smoking and sex and whether individual estimates predicted abstinence during the last week of the monitoring period and at the 2-month follow-up. Age, sex, and baseline nicotine dependence were entered into the equations as covariates.

\section{Methods}

\section{Participants}

A total of 272 Dutch daily smoking adolescents contacted the research team in response to advertisements and articles about the study that were published or displayed in newspapers, on Web sites, and in community centers. Upon contacting the researchers, interested candidates were sent a detailed study description. After reading the detailed information, 189 of the 272 candidates still wanted to participate and were assessed for eligibility through a telephone screening. To qualify, candidates had to be aged 15-19 years, smoke at least 1 cigarette/day, be highly motivated to quit, and not be currently enrolled in a cessation program. Participants aged 15 years had to have been smoking daily for at least 1 year to be eligible (this criterion did not apply for those aged 16-19 years). Four candidates were not accepted because they had already quit smoking and nine candidates withdrew before entering the study, leaving 176 adolescents who were enrolled officially. Of the 176 participants, 19 stopped completing assessments before or right after the quit day and 16 dropped out during the first postquit week. For these patterns of missing data, we concluded that they were not at random and therefore should be excluded from our analyses. Three additional subjects were excluded because they achieved 24-hr abstinence for the first time a week after the target quit day, which is problematic for the present analyses since the actual quit day was set to the target quit day. The final sample, thus, consisted of 138 subjects. In the logistic regression analysis, 12 subjects could not be included because they failed to return the baseline questionnaire and 4 subjects were excluded because their smoking during the last week could not be verified. Half of the 38 subjects who were excluded from the original sample of 176 individuals did not return the baseline questionnaire and, thus, were not included in the following attrition analysis. $t$ Tests indicated that those excluded were marginally more nicotine dependent, $t(136)=$ $2.04, p=.053$, and reported a higher average of number of cigarettes smoked per day at baseline, $t(136)=2.04, p=.015$, compared with those who were retained in the analyses. Excluded subjects did not seem to differ from those included according to age, sex, and whether they quit in the past 12 months.

The final sample $(N=138)$ consisted of 49 male $(35.5 \%)$ and 89 female $(64.5 \%)$ adolescents, who were receiving or had 
recently received regular education: $5.7 \%$ received lower vocational education, $39.7 \%$ received intermediate vocational education, $13.5 \%$ received high school education, $12.8 \%$ received preuniversity education, and $16.3 \%$ were in college $(12.1 \%$ unknown due to missing baseline questionnaire). Ages were distributed as follows: $15(2.2 \%), 16(32.4 \%), 17(30.1 \%), 18$ (15.4\%), 19 (18.4\%), and two persons had just turned 20 (1.5\%; $M=17.2$ years, $S D=1.2$ ). The average age at onset of daily smoking was 14.3 years $(S D=1.5)$, and the average length of time during which adolescents smoked daily was 2.9 years $(S D=1.6)$. Regarding quit attempts in the past 12 months, $14.5 \%$ had not tried to quit or cut down their smoking, 31.9\% had tried to quit smoking but not to cut down, $15.9 \%$ had tried to cut down their smoking but not to quit, and $29.0 \%$ had made attempts to cut down and quit smoking ( $8.7 \%$ unknown). Among those who reported having made a quit attempt, the average number of prior quit attempts in the past 12 months was $1.9(S D=1.2)$. Most participants smoked between 11 and 20 cigarettes/day.

\section{Procedure and design}

All 176 participants from the original sample were sent a baseline questionnaire, which was generally completed 1 week prior to the onset of the monitoring period. If needed, participants also received a letter for their schools that requested the school board's cooperation. For each individual, the period of monitoring always started on the first Monday following the telephone screening. Participants were monitored for 4 weeks: 1 week prior to the target quit day, during which time they were instructed to smoke ad libitum, and 3 weeks after the target quit day. For all participants, the assigned target quit day was the eighth day of the study.

During the monitoring period, participants were asked to respond to an Internet-based survey (on any desktop or laptop computer available) three times per day during the following intervals: in the morning (to be completed between 10 a.m. and noon), in the afternoon (3-5 p.m.), and in the evening (8-10 p.m.). Participants were not required to specify their location when they completed the assessments. The survey always contained the same questions and took about $3 \mathrm{~min}$ to complete. Printed paper diaries with identical questions were provided in case participants were unable to access the Internet; these were to be submitted online as soon as participants had access to the Internet again. Participants who failed to complete a questionnaire within the designated sampling window were sent a text message to remind them. Participants were not required to initiate reports of smoking or withdrawal symptoms outside the fixed intervals (event-contingent recording). Participants received the first instructions on the study during the telephone screening interview and instructions on the use of the Internetbased survey through E-mail. Participants were phoned by the research team twice during the course of the study to ask how they were doing and to ensure compliance. Two months after the end of the monitoring period, participants completed an online follow-up survey. Participants received remuneration of 40 Euros for completion of the baseline questionnaire and the 4 weeks of monitoring and an additional 10 Euros upon completion of the follow-up.

\section{Measures}

Withdrawal symptoms. We used 10 items from the Wisconsin Smoking Withdrawal Scale (WSWS) to determine adolescents' daily levels of the withdrawal symptoms craving, negative affect, and hunger (Welsch et al., 1999). The negative affect and hunger subscales include items that cover all symptoms of the smoking withdrawal syndrome as listed in Diagnostic and Statistical Manual of Mental Disorders, Fourth Edition (DSM-IV), except for insomnia. Although craving is not one of the DSM-IV criteria, it is considered to be an essential component of the abstinence withdrawal syndrome (e.g., Hughes, Higgins, \& Bickel, 1994) and was included in the daily assessments. The WSWS has good construct validity with high reliabilities for the three symptoms (West, Ussher, Evans, \& Rashid, 2006), and the shortened version of 10 items has been applied successfully in prior EMA research on smoking relapse (McCarthy et al., 2006). Two WSWS items were used to measure craving, six items to measure negative affect, and two items to measure hunger at each daily assessment. The 10 items could be answered on a Likert scale ranging from $1=$ strongly disagree to $5=$ strongly agree. A high score on the items, thus, represented high levels of withdrawal symptoms.

Prior to performing the analyses, we examined the factorial validity and internal validity of the three subscales of the WSWS. For each assessment $(28$ days $\times 3$ times a day $=84)$, we conducted a confirmatory factor analysis. The WSWS had a firm factorial validity with high principal loadings (mean values varied between .74 and .92) and an adequate model fit (mean fit values were $\chi^{2}(17)=34.54, p=.000$, comparative fit index $=$ .978 , root mean squared error of approximation $=.103)$. To determine the internal consistency of the withdrawal scales across all assessments, we computed alphas for each assessment and averaged these. This resulted in an average alpha of .88 $(S D=.04)$ for craving, $.75(S D=.04)$ for negative affect, and .66 $(S D=.09)$ for hunger. All three factors were intercorrelated: craving and negative affect: $r=.54$; craving and hunger: $r=.42$, and negative affect and hunger: $r=.31$.

Nicotine dependence. Nicotine dependence at baseline was assessed using a multidimensional measure of nicotine dependence for adolescents, which has good psychometric properties (Kleinjan et al., 2007). This composition was derived from the modified Fagerström Tolerance Questionnaire (Fagerström \& Schneider, 1989) and the Hooked on Nicotine Checklist (DiFranza et al., 2000). The combined 11 items of the three subscales included aspects of emotional and physical symptoms of dependence (irritation, anger, restlessness, and the like when abstaining or smoking less) and behavioral symptoms of nicotine dependence (e.g., intensity of smoking). The scale was composed with the standardized values (range $=1-4)$ since response categories were not the same for each item. Cronbach's alpha was .79. The average level of baseline nicotine dependence was $2.6(S D=0.48)$.

\section{Outcome variables}

For the present analyses, we examined abstinence during the last week of the study and at the 2-month follow-up. A dummy coded variable was constructed with 0 indicating that smoking occurred during the past 7 days of the monitoring period (even if only one cigarette) and 1 representing abstinence. Similarly, at follow-up, participants were asked whether they were currently abstinent, with response choices $1=$ "I have reverted to smoking as much as when I started the study," 2 = "I have cut back on smoking (compared to when I started the study)," and 3 = "I have quit smoking entirely." Response choices 1 and 2 were grouped and given the score 0 , which indicated that they had reverted to smoking, and 3 was recoded as 1 , representing abstinence. 


\section{Data analyses}

We used piecewise linear growth curve modeling (LGCM) in Mplus (Muthén \& Muthén, 1998-2006) to specify growth models of craving, negative affect, and hunger, containing an intercept as well as linear or quadratic terms during both prequit (Days 3-7) and postquit (Days 8-21) periods. The first two days of the week prior to the quit attempt (Days 1 and 2) were omitted from the growth curves because the withdrawal scores on those days were much higher relative to the other prequit days. This is a common issue in EMA studies since participants need to complete the questions a number of times before their responses are valid. Since the last week (Days 22-28) was used to determine one of the study outcomes (smoking during last week), the growth curves did not include this week. For those who did not achieve 24-hr abstinence on the target quit day, the actual quit day was set to Day 8. The three daily assessments were aggregated into daily averages.

Because the distributions of the symptoms were somewhat skewed and leptokurtic and assumed random missing values, we used the robust full information maximum likelihood estimator. In addition, a large proportion of the sample reported smoking after achieving abstinence $(68.8 \%$ in the first week after the target quit day, and $40.6 \%$ in the second week after the target quit day), which is likely to affect the growth curves. If we would compose different groups on the basis of their smoking after achieving 24-hr abstinence, the groups would be too small to perform LGCM, and statistical power would be jeopardized. To obtain some indication of how growth curves might differ as a function of post-abstinence smoking, we performed $t$ tests to compare the growth parameters of the postquit growth curves between the following two groups: (a) those who did not smoke on any day during the second study week (which was the first week after cessation) and (b) those who smoked on at least 1 day during that week. We performed similar $t$ tests for groups that were distinguished on the basis of their smoking in the third week of the study. Differences between boys and girls in prequit and postquit estimated means were tested as well.

Next, logistic regression analyses were conducted to test whether individual estimates of symptom trajectories (prequit and postquit intercepts, slopes, and quadratic terms) predicted abstinence during the last week of the monitoring period and abstinence at follow-up. Since slopes and quadratic terms automatically have very low SDs, odds ratios (ORs) obtained in the logistic regression analyses are likely to be excessively large. We avoided this problem by using standardized values of the growth curve estimates in the logistic regression analyses. Finally, age, sex, and nicotine dependence were included as covariates for both outcomes.

\section{Results}

Although participants were instructed to smoke ad libitum during the first week of monitoring, eight participants (5.8\%) quit smoking before the target quit day. The majority of participants reached $24-\mathrm{hr}$ abstinence on the target quit day (73.2\%) and $14.5 \%$ on the next day. The remaining participants $(4.3 \%)$ reached 24-hr abstinence between Days 10 and 12.

\section{Latent growth curve analysis}

We first examined whether a linear or quadratic trend best fit the data. All prequit intercepts deviated significantly from zero (Table 1), but the prequit slopes were not significant, as can be seen in Figure 1, which depicts the best fitting growth curves.

For postquit craving and hunger, a quadratic model described the data best, with intercepts that deviated significantly from zero and with significant negative slopes and positive quadratic terms. For the course of postquit negative affect, a linear function was most suitable, with a significant intercept and significantly declining slope. The model fit indices evidenced good to excellent fit of the models (see Table 1).

Translated to more descriptive terms, we can say that prequit withdrawal levels were highly stable and that, on Day 8, withdrawal symptoms (especially craving) increased substantially. A strong decrease in symptoms was visible during the week following the quit day. During the 2 weeks after the target quit day, negative affect and hunger decreased monotonically to a level comparable with the beginning of Day 8 , and craving showed a steeper decrease. As can be seen from Figure 1, the three symptoms followed the same overall pattern, although craving was most salient in its elevation on the quit day and its curvature.

We also tested whether the growth curve parameters differed as a function of post-abstinence smoking and sex (only the significant results are reported). Prequit intercepts of craving were significantly higher among those who smoked during the third study week compared with those who were abstinent that entire week, $t(120)=-2.23, p=.027$. In addition, those who smoked during the third study week displayed marginally stronger negative slopes for hunger, $t(120)=1.91, p=.058$, and stronger quadratic estimates for hunger, $t(120)=-1.98, p=.050$. As for differences between sexes, girls had on average a marginally higher postquit intercept of negative affect, $t(120)=-1.86, p=.066$.

\section{Predicting abstinence}

Among the subsample of 122 subjects, $51.6 \%$ were verified to be abstinent during the last study week. At follow-up, 32.5\% reported current abstinence. Age, sex, and nicotine dependence were not significantly related to abstinence during the last week or with abstinence at follow-up (Table 2).

Of all individual growth curve parameters, only the prequit and postquit intercepts of craving and the postquit slope and quadratic term of hunger predicted abstinence during the last study week. Higher intercepts of prequit craving, or a higher general craving level across all prequit days, decreased the odds for abstinence during the last week. For hunger, a lower slope and higher quadratic term predicted failure of abstinence during the last week. This means that those who reverted to their prequit levels of hunger fastest were less likely to be abstinent later on. We found no effects of growth curves estimates on abstinence at the 2-month follow-up.

\section{Discussion}

The main objective of the present study was to describe withinperson variability in withdrawal symptoms and its association with smoking cessation in a sample of daily smoking adolescents who embarked on a serious quit attempt. All withdrawal symptoms 
Nicotine withdrawal symptoms in adolescents

Table 1. Intercepts, slopes, and model fit indices of craving, negative affect, and hunger $(N=138)$

\begin{tabular}{|c|c|c|c|c|c|c|c|}
\hline & $b$ & $S E$ & $Z$ & $p$ & $\chi^{2}(295)$ & Comparative fit index & $\begin{array}{l}\text { root mean squared error } \\
\text { of approximation }\end{array}$ \\
\hline Craving-prequit & & & & & 160.12 & 1.00 & .000 \\
\hline Intercept & 2.319 & .083 & 27.95 & .000 & & & \\
\hline Slope & .011 & .023 & 0.46 & .649 & & & \\
\hline \multicolumn{8}{|l|}{ Craving-postquit } \\
\hline Intercept & 3.053 & .098 & 31.13 & .000 & & & \\
\hline Slope & -.140 & .021 & -6.57 & .000 & & & \\
\hline Quadratic term & .007 & .002 & 4.58 & .000 & & & \\
\hline Negative affect-prequit & & & & & 214.18 & .958 & .045 \\
\hline Intercept & 1.412 & .044 & 31.92 & .000 & & & \\
\hline Slope & .018 & .014 & 1.34 & .179 & & & \\
\hline \multicolumn{8}{|l|}{ Negative affect-postquit } \\
\hline Intercept & 1.662 & .055 & 30.17 & .000 & & & \\
\hline Slope & -.014 & .004 & -3.80 & .000 & & & \\
\hline Hunger-prequit & & & & & 198.30 & .974 & .040 \\
\hline Intercept & 1.737 & .060 & 29.17 & .000 & & & \\
\hline Slope & .017 & .016 & 1.04 & .297 & & & \\
\hline \multicolumn{8}{|l|}{ Hunger-postquit } \\
\hline Intercept & 2.179 & .086 & 25.32 & .000 & & & \\
\hline Slope & -.057 & .017 & -3.32 & .001 & & & \\
\hline Quadratic term & .003 & .001 & 2.32 & .020 & & & \\
\hline
\end{tabular}

(craving, negative affect, and hunger) increased on the designated quit day. The course over time for craving and hunger were best described by a quadratic term, and a linear model best suited negative affect. Within 2 weeks postquit, both abstinent and relapsed adolescents had reverted to levels comparable with those during the prequit period. Higher levels of craving during the prequit week and on the target quit day (intercepts) decreased the odds of being abstinent during the last week of the study. The prequit and postquit slopes of craving did not predict abstinence during the last week. Growth parameters of negative affect were not associated with chances of being abstinent. For hunger, it appeared that those who reverted to their prequit levels of hunger fastest were less

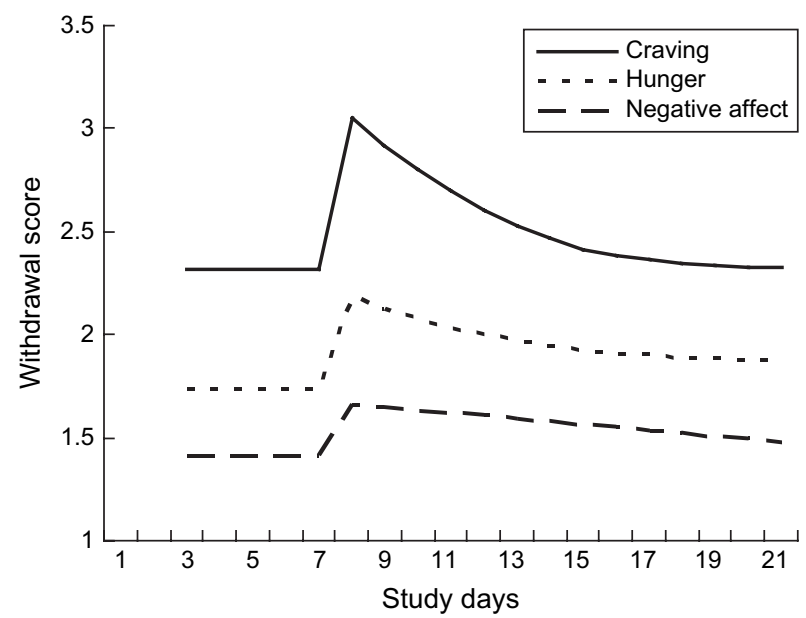

Figure 1. Estimated growth curves of craving, hunger, and negative affect. likely to be abstinent during the last study week. Finally, among all three symptoms, none of the growth model characteristics predicted abstinence at the 2-month follow-up.

The elevation of all three withdrawal symptoms on the designated quit day is in line with findings among adults (Hughes, 1992; Jorenby et al., 1996; McCarthy et al., 2006; Piasecki et al., 1998; for an exception, see Shiffman et al., 1997) and with preliminary results among adolescents (Smith et al., 2008). Craving appeared to be the most salient symptom in its elevation and curvature, which is in accordance with the study on adolescents by Smith et al. (2008). It is also in line with the consistent report by adolescents of craving being the most salient and severe symptom in general (Colby, Tiffany, Shiffman, \& Niaura, 2000).

A comparison of the craving growth curve from the present study with the one among adults as reported by McCarthy et al. (2006), who used an identical craving scale, shows that both adult and adolescent craving levels remained quite stable during the prequit period but that adolescents seemed to revert to their baseline craving levels more quickly. It seems plausible that those who reverted to smoking after achieving abstinence experienced relief of craving, which may account for the relatively quick overall decline. However, given that the postquit slopes did not differ between those who reported postquit smoking and those who did, it seems that adolescents who successfully quit were not bothered by elevated craving for long. This may explain why the rate of decline did not predict abstinence.

It is interesting that both prequit and postquit intercepts of craving had a significant effect on abstinence during the last study week, whereas the shape of the course over time did not. Thus, for craving, this seems to suggest that how adolescents enter the quitting process is more important than the process itself. This 


\begin{tabular}{|c|c|c|c|c|}
\hline & \multicolumn{2}{|c|}{ Smoking during last week $(N=122)$} & \multicolumn{2}{|c|}{ Smoking status at follow-up $(N=126)$} \\
\hline & Odds ratio & $95 \% C I$ & Odds ratio & $95 \% C I$ \\
\hline Age & 1.34 & $.97-1.85$ & .86 & $.61-1.21$ \\
\hline Sex & 1.35 & $.63-2.91$ & 1.61 & $.69-3.78$ \\
\hline Nicotine dependence & .72 & $.33-1.59$ & 1.04 & $.45-2.43$ \\
\hline \multicolumn{5}{|l|}{ Craving-prequit } \\
\hline Intercept & $.63^{*}$ & $.42-.94$ & .91 & $.59-1.40$ \\
\hline Slope & .90 & $.60-1.33$ & 1.11 & $.74-1.67$ \\
\hline \multicolumn{5}{|l|}{ Craving_postquit } \\
\hline Intercept & $.59^{*}$ & $.38-.91$ & .69 & $.42-1.12$ \\
\hline Slope & .90 & $.60-1.35$ & .66 & $.42-1.05$ \\
\hline Quadratic term & .98 & $.67-1.44$ & 1.27 & $.83-1.94$ \\
\hline \multicolumn{5}{|c|}{ Negative affect—prequit } \\
\hline Intercept & .93 & $.62-1.40$ & .85 & $.54-1.35$ \\
\hline Slope & .76 & $.50-1.14$ & 1.22 & $.81-1.84$ \\
\hline \multicolumn{5}{|c|}{ Negative affect—postquit } \\
\hline Intercept & .76 & $.45-1.25$ & .64 & $.34-1.18$ \\
\hline Slope & .90 & $.57-1.41$ & .60 & $.34-1.08$ \\
\hline \multicolumn{5}{|l|}{ Hunger-prequit } \\
\hline Intercept & .82 & $.57-1.20$ & 1.19 & $.80-1.76$ \\
\hline Slope & .99 & $.68-1.45$ & 1.43 & $.93-2.21$ \\
\hline \multicolumn{5}{|l|}{ Hunger-postquit } \\
\hline Intercept & 1.07 & $.69-1.66$ & 1.13 & $.72-1.79$ \\
\hline Slope & $1.68^{\star}$ & $1.03-2.72$ & 1.12 & $.73-1.73$ \\
\hline Quadratic term & $.63^{*}$ & $.41-.97$ & .90 & $.60-1.36$ \\
\hline
\end{tabular}

Note. The estimates for the effects of intercepts, slopes, and quadratic terms are from the multivariate analyses in which age, sex, and nicotine dependence were included as covariates. Prequit and postquit predictors were not included in the analyses simultaneously. ${ }^{\star} p<.05$.

explanation is contradicted somewhat by our findings that baseline nicotine dependence did not predict abstinence. It is possible that an elevation in symptoms had already taken place in the 1 week between completion of the baseline questionnaire and the start of the monitoring period. Since the prequit period was very short (7 days, of which only 5 were included in the growth curves), the present effects of craving intercepts might reflect anticipatory mechanisms (cf., McCarthy et al., 2006). Taking this one step further, it may be less crucial to target craving once 24 -hr abstinence is achieved but is rather essential to decrease craving levels before the attempt is started. Although nicotine replacement therapy (NRT) has been found to reduce levels of craving among adults (Hughes, Shiffman, Callas, \& Zhang, 2003), prior studies have found little support for the efficacy of NRT among adolescents (Hanson, Allen, Jensen, \& Hatsukami, 2003; Killen et al., 2004; Moolchan et al., 2005). More research is needed to examine how NRT could be improved and to explore alternative treatments to decrease prequit craving among adolescents.

The target quit day peak in negative affect was less pronounced than that of craving, and the prequit and postquit intercepts and slopes of negative affect did not predict treatment outcomes. The observation that levels of both prequit and postquit negative affect were relatively low and showed only a modest elevation is in line with prior findings among adolescents (Smith et al., 2008). However, the postquit part of the growth curve for negative affect did show discrepancies from that among adults (McCarthy et al., 2006).
Whereas adults' levels of negative affect remained stable across the 3 weeks after cessation (the slope coefficient was positive but not significant), the postquit slope among adolescents was significant and negative. Thus, as with craving, adolescents seem to revert to their baseline levels of negative affect more quickly than adults. Apparently, trying to quit does not instigate intense negative affect among adolescents, and the elevation and pace of the subsequent decrease in symptoms do not seem to provoke relapse either. This may be the result of adolescents in general being subject to more variable and intense moods and more anxiety than young children and adults (Buchanan et al., 1992). Alternatively, although moods may be more variable and intense during adolescence, those predisposed to depressive feelings may be more sensitive to mood changes, and changes in negative affect may be linked more closely to abstinence among adolescents vulnerable to depressive mood.

Prequit levels of hunger were low and stable, and they resembled prequit levels of hunger among adults (McCarthy et al., 2006). Although adults and adolescents seem to experience a similar modest increase in hunger during the quit day, the postquit course over time appears to be different. Whereas the postquit slope among adults showed a marginally significant linear increase over time, the trend among adolescents was a significant decline and was quadratic. However, this difference in results might be explained by postquit smoking since those who had been smoking during the third study week displayed stronger quadratic terms for hunger. In other words, those who had reverted to smoking 
experienced faster declines in feelings of hunger. The latter finding also provides an explanation of why the quadratic term of hunger predicted abstinence during the last week since those who smoked during the third week were more likely to be smoking during the fourth week as well.

Previous findings by Smith et al. (2008) indicated that the course of withdrawal over time among adolescents who achieved abstinence differed for boys and girls, but we found little evidence of such relationship. One exception was that girls had marginally higher postquit intercepts of negative affect than boys, which is plausible considering that the literature consistently indicates that females are more vulnerable to depressive symptoms (Piccinelli \& Wilkinson, 2000), and this also applies to Dutch adolescents (Engels, Finkenauer, Meeus, \& Dekovic, 2001). Nonetheless, girls did not seem to be at additional risk since the intercepts of negative effect were not associated with cessation outcomes, and sex as an independent covariate did not predict abstinence.

In sum, the findings generally suggest that an adolescent's ability to quit smoking successfully does not depend a great deal on how withdrawal symptoms evolve over time after achieving abstinence. We should be cautious, however, in declaring withdrawal symptoms to be less important to the adolescent cessation process. First, the present findings do not indicate whether day-to-day variations in withdrawal might predict lapse or relapse the next day (as has been demonstrated for craving among adults; Shiffman, Paty, Gwaltney, \& Dang, 2004). Up to now, almost no research has been devoted to the dynamic effects of withdrawal symptoms among adolescent smokers who are in the midst of a quit attempt (except for an exploratory study among 13 adolescents by Gwaltney, Bartolomei, Colby, \& Kahler, 2008). Second, in interpreting the present results, we must recognize several study limitations. For instance, participants who were categorized as abstinent during the last week of the monitoring period may have been identified as smokers by other assessments. The likelihood that this occurred is restricted since compliance was high and only $7.1 \%$ of postquit assessments (not days) were not completed, but it remains a possibility.

Furthermore, not all participants managed to achieve 24-hr abstinence on the designated quit day. Even though each person's actual quit day was set to Day 8 , and the postquit growth models started with the actual quit day for each person, those who did not quit until after the target quit day may have differed in initial levels of withdrawal from the ones who succeeded. Those who quit later continued to smoke between the target quit day and their actual quit day, which is likely to influence the course of withdrawal.

We also must consider a possible reactivity effect associated with the intensive self-monitoring that is a core component of EMA research designs. Intensive self-monitoring has been argued to reduce the problem behavior that is being targeted to be changed (Shiffman et al., 2008). This may occur particularly when people want to change their behavior and are able to exert control over it, which is the case for smoking cessation. Although studies have found little support for the concern that EMA produces significant reactivity (Shiffman et al., 2008), it remains possible that the intensive self-monitoring influenced the way adolescents dealt with withdrawal symptoms and that it contributed to the rates of abstinence found in the present study. We must keep this possibility in mind since abstinence rates were remarkably high (51.6\% during the last study week and 32.5\% at the 2-month follow-up) compared with other studies among adolescents (O'Connell et al., 2004; Sussman, 2002).

Alternatively, the high abstinence rates could have been a result of attrition. Those who dropped out or who were excluded from the present analyses had significantly higher levels of baseline daily smoking and were more dependent on nicotine. Although higher levels of nicotine dependence did not affect cessation outcomes, those who drop out of studies in which smoking abstinence needs to be achieved often can be considered unsuccessful quitters (e.g., Smith et al., 2008). Our ability to generalize the present results to all daily smoking adolescents may be restricted somewhat. These limitations notwithstanding, the present study has revealed new insights into the natural history of the course of withdrawal symptoms over time and the association of this course with cessation outcomes in adolescents.

\section{Funding}

This research was supported by a grant from the Dutch Asthma Foundation and a fellowship grant to Rutger Engels from the Netherlands Organization of Scientific Research.

\section{Declaration of Interests}

None declared.

\section{Acknowledgments}

The research presented in the present paper was carried out at the Behavioral Science Institute, Radboud University Nijmegen, The Netherlands.

\section{References}

Beyth-Marom, R., Fischhoff, B., Jacobs-Quadrel, M., \& Furby, L. (1991). Teaching decision making to adolescents: A critical review. In J. Baron \& R. V. Brown (eds.), Teaching decision making to adolescents (pp. 19-61), Hillsdale, NJ: Lawrence Erlbaum Associates.

Buchanan, C. M., Eccles, J. S., \& Becker, J. B. (1992). Are adolescents the victims of raging hormones: Evidence for activational effects of hormones on moods and behaviour at adolescence. Psychological Bulletin, 111, 62-107.

Chambers, R. A., Taylor, J. R., \& Potenza, M. N. (2003). Developmental neurocircuitry of motivation in adolescence: A critical period of addiction vulnerability. American Journal of Psychiatry, $160,1041-1052$.

Colby, S. M., Tiffany, S. T., Shiffman, S., \& Niaura, R. S. (2000). Are adolescent smokers dependent on nicotine? A review of the evidence. Drug and Alcohol Dependence, 59, 83-95.

DiFranza, J. R., Rigotti, N. A., McNeill, A. D., Ockene, J. K., Savageau, J. K., St Cyr, D., et al. (2000). Initial symptoms of nicotine dependence in adolescents. Tobacco Control, 9, 313-319.

Engels, R. C. M. E., Finkenauer, C., Meeus, W., \& Dekovic, M. (2001). Parental attachment and adolescents' emotional 
adjustment: The role of interpersonal tasks and social competence. Journal of Counseling Psychology, 48, 428-439.

Fagerström, K. O., \& Schneider, N. G. (1989). Measuring nicotine dependence: A review of the Fagerström Tolerance Questionnaire. Journal of Behavioral Medicine, 12, 159-182.

Garrison, M. M., Christakis, D. A., Ebel, B. E., Wiehe, S. E., \& Rivara, F. P. (2003). Smoking cessation interventions for adolescents: A systematic review. American Journal of Preventive Medicine, 25, 363-367.

Gwaltney, C. J., Bartolomei, R., Colby, S. M., \& Kahler, C. W. (2008). Ecological Momentary assessment of adolescent smoking cessation: A feasibility study. Nicotine \& Tobacco Research, $10,1185-1190$.

Hanson, K., Allen, S., Jensen, S., \& Hatsukami, D. (2003). Treatment of adolescent smokers with the nicotine patch. Nicotine \& Tobacco Research, 5, 515-526.

Hughes, J. R. (1992). Tobacco withdrawal in self-quitters. Journal of Consulting and Clinical Psychology, 60, 689-697.

Hughes, J. R. (2007). R. Measurement of the effects of abstinence from tobacco: A qualitative review. Psychology of Addictive Behaviors, 21, 127-37.

Hughes, J. R., Higgins, S. T., \& Bickel, W. K. (1994). Nicotine withdrawal versus other drug withdrawal syndromes: Similarities and dissimilarities. Addiction, 89, 1461-1470.

Hughes, J. R., Shiffman, S., Callas, P., \& Zhang, J. (2003). A metaanalysis of the efficacy of over-the-counter nicotine replacement. Tobacco Control, 12, 21-27.

Jorenby, D. E., Hatsukami, D. K., Smith, S. S., Fiore, M. C., Allen, S., Jensen, J., et al. (1996). Characterization of tobacco withdrawal symptoms: Transdermal nicotine reduces hunger and weight gain. Psychopharmacology, 128, 130-138.

Kassel, J. D., Stroud, L. R., \& Paronis, C. A. (2003). Smoking, stress, and negative affect: Correlation, causation, and context across stages of smoking. Psychological Bulletin, 129, 270-304.

Killen, J. D., Robinson, T. N., Ammerman, S., Hayward, C., Rogers, J., Stone, C., et al. (2004). Randomized clinical trial of the efficacy of bupropion combined with nicotine patch in the treatment of adolescent smokers. Journal of Consulting and Clinical Psychology, 72, 729-735.

Kleinjan, M., Van Den Eijnden, R. J. J. M., Van Leeuwe, J., Brug, J., Otten, R., \& Engels, R. C. M. E. (2007). Factorial and convergent validity of nicotine dependence measures in adolescents: Comparing one- and multidimensional approaches. Nicotine \& Tobacco Research, 9, 1-10.

Leatherdale, S. T. (2006). School-based smoking cessation programs: Do youth smokers want to participate in these programs? Addictive Behaviors, 31, 1449-1453.

McCarthy, D. E., Piasecki, T. M., Fiore, M. C., \& Baker, T. B. (2006). Life before and after quitting smoking: An electronic diary study. Journal of Abnormal Psychology, 115, 454-466.
Mermelstein, R. (2003). Teen smoking cessation. Tobacco Control, $12,25-34$.

Moolchan, E. T., Robinson, M. L., Ernst, M., Cadet, J. L., Pickworth, W. B., Heishman, S. J., et al. (2005). Safety and efficacy of the nicotine patch and gum for the treatment of adolescent tobacco addiction. Pediatrics, 115, e407-e414.

Muthén, L. K., \& Muthén, B. O. (1998-2006). Mplus user's guide (4th ed.). Los Angeles: Author.

O'Connell, M. L., Freeman, M., Jennings, G., Chan, W., Greci, L. S., Manta, I. D., et al. (2004). Smoking cessation for high school students impacts evaluation of a novel program. Behaviour Modification, 28, 133-146.

Piasecki, T. M., Fiore, M. C., \& Baker, T. B. (1998). Profiles in discouragement: Two studies of variability in the time course of smoking withdrawal symptoms. Journal of Abnormal Psychology, 107, 238-251.

Piccinelli, M., \& Wilkinson, G. (2000). Gender differences in depression: Critical review. British Journal of Psychiatry, 177, 486-492.

Prokhorov, A. V., Hudmon, K. S., Cinciripini, P. M., \& Marani, S. (2005). "Withdrawal symptoms" in adolescents: A comparison of former smokers and never-smoker. Nicotine \& Tobacco Research, 7, 909-913.

Shiffman, S., Engberg, J., Paty, J. A., Perz, W., Gnys, M., Kassel, J. D., et al. (1997). A day at a time: Predicting smoking lapse from daily urge. Journal of Abnormal Psychology, 106, 104-116.

Shiffman, S., Paty, J. A., Gwaltney, C. J., \& Dang, Q. (2004). Immediate antecedents of cigarette smoking: An analysis of unrestricted smoking patterns. Journal of Abnormal Psychology, 113, 166-171.

Shiffman, S., Stone, A. A., \& Hufford, M. R. (2008). Ecological momentary assessment. Annual Review of Clinical Psychology, 4, $1-32$.

Smith, A. E., Cavallo, D. A., McFetridge, A., Liss, T., \& KrishnanSarin, S. (2008). Preliminary examination of tobacco withdrawal in adolescent smokers during smoking cessation treatment. Nicotine \& Tobacco Research, 10, 153-159.

Steinberg, L., \& Cauffman, E. (1996). Maturity of judgment in adolescence: Psychosocial factors in adolescent decision making. Law and Human Behavior, 20, 249-272.

Sussman, S. (2002). Effects of sixty six adolescent tobacco use cessation trials and seventeen prospective studies of self-initiated quitting. Tobacco Induced Diseases, 1, 35-81.

Welsch, S. K., Smith, S. S., Wetter, D. W., Jorenby, D. E., Fiore, M. C., \& Baker, T. B. (1999). Development and validation of the Wisconsin Smoking Withdrawal Scale. Experimental and Clinical Psychopharmacology, 7, 354-361.

West, R., Ussher, M., Evans, M., \& Rashid, M. (2006). Assessing DSM-IV nicotine withdrawal symptoms: A comparison and evaluation of five different scales. Psychopharmacology, 184, 619-627. 